\title{
Molecular QSO Absorption Line Systems
}

\author{
C. L. Carilli
}

National Radio Astronomy Observatory, Socorro, NM, USA 87801

\author{
K. M. Menten \\ Max-Planck-Institut für Radioastronomie, Auf dem Hügel 69, D-53121 \\ Bonn, Germany
}

\begin{abstract}
We review observations of molecular absorption line systems at high redshift toward red quasars and gravitational lenses.
\end{abstract}

The steeply falling power law column density distribution function of QSO absorption line systems implies that for every 1000 Ly $\alpha$ forest lines from absorbers with hydrogen column density, $N_{\mathbf{H}}<10^{15} \mathrm{~cm}^{-2}$ there will be only one system with $N_{\mathrm{H}} \geq 10^{22} \mathrm{~cm}^{-2}$ (Hu et al. 1995). Although rare, these extreme high column density systems provide the most direct and detailed probes of the dense, pre-star-forming interstellar medium (ISM) in galaxies at substantial look-back times. Unfortunately, such high column densities also lead to substantial optical dust extinction, e.g. for a normal dust-to-gas ratio, $A_{V}>6$ for $N_{\mathrm{H}}>10^{22} \mathrm{~cm}^{-2}$; hence finding such absorbers using optical spectroscopy is problematic. One method used for finding these systems is to search for molecular and $\mathrm{HI} 21 \mathrm{~cm}$ absorption toward flat spectrum radio sources with red (or absent) optical counterparts. Four high redshift molecular absorption line systems have been discovered this way, in two of which the absorption occurs in a gravitational lens $(0218+357$ at $z=0.685$ and $1830-211$ at $z=0.886$; Wiklind $\&$ Combes 1995, 1996a). In the other two systems $(1413+135$ at $z=0.247$ and $1504+357$ at $z=0.673$ ) the absorption takes place in the host galaxy of an AGN (Wiklind \& Combes 1994, 1996b).

So far, 15 different molecules have been identified in these redshifted systems, plus many of their isotopomers, including complex, multi-atom molecules such as the cyclic species $\mathrm{C}_{3} \mathrm{H}_{2}$ (Combes \& Wiklind 1999; Menten et al. 1999). The lines can be relatively broad, as for $1504+377$ with a FWHM $=100 \mathrm{~km}$ $\mathrm{s}^{-1}$, or extremely narrow, as for $1413+135\left(\mathrm{FWHM}=1 \mathrm{~km} \mathrm{~s}^{-1}\right)$. As opposed to emission studies, observations of redshifted absorption provide the ability to probe very narrow pencil beams through the intervening galaxy and, thus, given a sufficiently strong background source, to determine the physical and chemical conditions in single molecular clouds by observations of rare molecular species.

The best studied of the high- $z$ molecular absorption line sources in terms of high resolution imaging is that associated with the gravitational lens system 1830-211 (Wiklind \& Combes 1996a). At its redshift of 0.89, many of the ground level rotational transitions of molecules commonly found in galactic molecular clouds redshift into the observing bands of the VLA and the VLBA (Carilli et al. 1997, 1998; Menten et al. 1999; see Fig. 1). Strong absorption is seen 

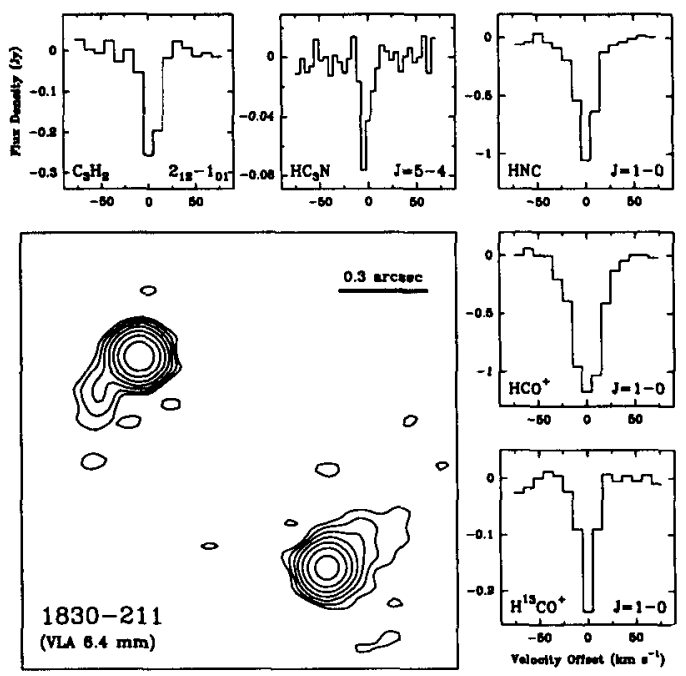

Figure 1. The contour plot shows an image of the 'Einstein ring' radio source PKS $1830-211$ at $43 \mathrm{GHz}$ with a resolution of $0.1^{\prime \prime}$ made with the Very Large Array. The spectra show molecular absorption by gas in the lensing galaxy, as observed with the Very Large Array toward the southwest radio component. Zero velocity corresponds to a heliocentric redshift of 0.88582 .

toward the SW radio component at $z=0.88582$; e.g, the HCN $J=1-0$ line has a peak opacity of 2.5 and FWHM $=25 \mathrm{~km} \mathrm{~s}^{-1}$. The limit of 0.3 to the opacity toward the "tail" of the SW component implies an upper limit to the cloud size of $800 \mathrm{pc}$ for $H_{0}=75 \mathrm{~km} \mathrm{~s}^{-1} \mathrm{Mpc}^{-1}, q_{0}=0.5$. A VLBA image of the SW component at $24 \mathrm{GHz}$ shows a core-jet structure extending $\approx 2$ mas to the northwest. Spectra of redshifted $\mathrm{HC}_{3} \mathrm{~N} J=5-4$ absorption at this resolution imply a lower limit to the cloud size of 2.5 mas, corresponding to $13 \mathrm{pc}$, although there may be sub-structure on scales of a few pc (Carilli et al. 1997, 1998). The implied upper limit to the volume averaged density is $1000 \mathrm{~cm}^{-3}$, and the lower limit to the molecular mass is $3 \times 10^{4} M_{\odot}$. This is consistent with a lower limit to the cloud size of $0.3 \mathrm{pc}$ set by the fact that the line excitation temperatures are comparable to the microwave background (Frye et al. 1997).

Toward the NE component of 1830-211 weak molecular absorption is detected at a velocity of $-146 \mathrm{~km} \mathrm{~s}^{-1}$, where zero velocity is defined by the redshift $(z=0.88582)$ of the absorption seen toward the SW component. Here the HCN $(1-0)$ optical depth is $\approx 50$ times smaller than for the zero velocity component observed toward the SW image (Wiklind \& Combes 1998; Carilli et al. 1998). Chengalur et al. (1999) have detected both $\mathrm{HI}$ and $\mathrm{OH}$ absorption toward 1830-211. Interestingly, the $\mathrm{HI}$ absorption is stronger at $-146 \mathrm{~km} \mathrm{~s}^{-1}$ than at $0 \mathrm{~km} \mathrm{~s}^{-1}$, implying an [HI/HCN] ratio differing by a factor of 125 between the two lines-of-sight, assuming equal spin temperature. 
Menten et al. (1999) conclude that the molecular abundance ratios for the 1830-211 absorber are similar to values found for Galactic dark clouds and do not show the high abundances of $\mathrm{HCO}^{+}, \mathrm{HCN}$, and $\mathrm{H}_{2} \mathrm{CO}$ relative to $\mathrm{CO}$ found in many diffuse clouds (Liszt \& Lucas 1995). Moreover, they find $\left[\mathrm{C}^{12} / \mathrm{C}^{13}\right]=$ 35 , which is smaller by a factor of two than the value measured in the Solar System and the local ISM, but comparable to that found in some inner Galaxy clouds. Since ${ }^{13} \mathrm{C}$ is only produced in low and intermediate mass stars, while ${ }^{12} \mathrm{C}$ is also produced in massive stars, this ratio is expected to decrease in time and with increasing stellar processing (Wilson \& Matteucci 1992). Studying the deuterium chemistry of the 1830-211 absorber, Shah et al. (1999) set an upper limit of 0.003 on the $[\mathrm{DCN} / \mathrm{HCN}]$ ratio. This is in the upper range of values seen in Galactic dense clouds, where the deuterium abundance in the molecular phase is greatly enhanced due to fractionation. Gerin \& Roueff (1999) state that this DCN upper limit is barely compatible with a high gas phase deuterium abundance of $10^{-4}$, perhaps indicating significant astration (Shah et al. 1999). A review of oxygen chemistry in $0218+357$ is given in Combes \& Wiklind (1999).

Overall, from detailed chemical modeling Gerin \& Roueff (1999) conclude that the high column density, low density, low $\mathrm{O}_{2}$ and DCN abundance, and high $\mathrm{CCH}$ abundance can be understood if the absorbing material is chemically in the High Ionization Phase (HIP), in which the fractional ionization is $\sim 10^{-6}$, the gas phase $\mathrm{C}$ abundance is high, and the chemistry is driven by charge transfer reactions with $\mathrm{H}^{+}$. The HIP is the sole stable phase for molecular clouds at low densities $\left(<10^{4} \mathrm{~cm}^{-3}\right)$.

The two known absorption systems associated with lensing galaxies in gravitational lens systems can be used to study the lens parameters. Wiklind \& Combes (1998) and Combes \& Wiklind (1999) discuss the potential of using single dish observations of the variation of molecular absorption line optical depths to measure the geometric time delay for $1830-211$.

The observed difference in absorption velocities between the SW and NE images in 1830-211 allows for tight constraints on the lens mass distribution and, thus, lensing models. Wiklind \& Combes $(1998)$ derive $V_{0} \approx 220 \sqrt{D} \mathrm{~km} \mathrm{~s}^{-1}$ for the rotation velocity, $V_{0}$, of the $z \approx 0.89$ lensing galaxy, with the "effective distance" $D$, in Gpc, in standard notation. Using the source redshift of 2.507 yields $V_{0} \approx 366 \mathrm{~km} \mathrm{~s}^{-1}$, implying a massive early-type spiral lens. The centroid position, inclination angle, and orientation of the lens in this model are consistent with the observed values in HST near-IR imaging (Lehar et al. 2000).

An interesting use of redshifted molecular absorption lines is to constrain the evolution of the temperature of the microwave background radiation. The critical densities for collisional excitation of the lower rotational lines of high dipole moment molecules such as $\mathrm{HC}_{3} \mathrm{~N}$ are typically $\geq 10^{5} \mathrm{~cm}^{-3}$. As the absorbing clouds seen toward 1830-211 are likely to have a lower density than this, one expects the molecular excitation for such molecules to be determined by the ambient radiation field, which, at $z=0.9$, will be dominated by the microwave background radiation, at least outside of active star forming regions. Combes \& Wiklind (1999) summarize single dish measurements of the excitation temperature for a number of molecules at $z=0.88582$ toward 1830-211, while Menten et al. (1999) present perhaps the most accurate measurement based on VLA observations of the $J=3-2$ and 5-4 transitions of $\mathrm{HC}_{3} \mathrm{~N}$, from which they 
derive $T_{e x}=4.5_{-0.6}^{+1.5}$. This is consistent with the expected microwave background temperature of $5.14 \mathrm{~K}$.

Drinkwater et al. (1998) and Wiklind \& Combes (1998) show how a comparison of redshifts derived from molecular absorption to those derived from $\mathrm{HI}$ $21 \mathrm{~cm}$ absorption constrains the cosmic evolution of the fine structure constant, $\alpha$. The current limits are not set by the accuracy of the measurements but by (possible) relative systematic motions of molecular and atomic absorbing clouds in galaxies. Most recently, Carilli et al. (2000) have used VLBI observations to constrain sight-lines to sub-kpc scales, thereby removing at least one of the uncertainties in the problem. They set a limit of $\left|\frac{\dot{\alpha}}{\alpha}\right|<3.5 \times 10^{-15}$ year $^{-1}$ to a look-back time of $4.8 \mathrm{Gyr}$, assuming that the velocity error is dictated by small scale ISM motions.

Acknowledgments. The National Radio Astronomy Observatory is a facility of the National Science Foundation, operated under cooperative agreement by Associated Universities, Inc.

\section{References}

Carilli, C.L. et al. 1997, in Structure and Evolution of the IGM, eds. Petitjean and Charlot (Editions Frontiers: Paris), p. 325

Carilli, C.L. et al. 1998, in Radio Emission from Galactic and Extragalactic Compact Sources, eds. Zensus et al. (ASP: San Francisco), p. 317

Carilli, C.L. et al. 2000, Phys. Rev. Lett., in press

Chengalur, J.N., de Bruyn, A.G., and Narasimha, D. 1999, A\&A, 343, L79

Combes, F., Wiklind T. 1999, in Highly Redshifted Radio Lines, eds. Carilli et al. (ASP: San Francisco), p. 210

Drinkwater, M.J. et al. 1998, MNRAS, 295, 457

Frye B., Welch W. J., Broadhurst T.: 1997, ApJ, 478, L25

Gerin, M., Roueff, E. 1999, in Highly Redshifted Radio Lines, eds. Carilli, et al. (ASP: San Francisco), p. 196

$\mathrm{Hu}$, E.M. et al. 1995, AJ, 110, 1526

Lehar, J. et al. $2000 \mathrm{ApJ}, 536,584$

Liszt H.S., Lucas R. 1995, A\&A, 299, 847

Menten, K.M., Carilli, C.L., and Reid, M.J. 1999, in Highly Redshifted Radio Lines, eds. Carilli et al. (ASP: San Francisco), p. 218

Shah, R. et al. 1999, in Highly Redshifted Radio Lines, eds. Carilli et al. (ASP: San Francisco), p. 233

Wiklind, T., Combes, F. 1994, A\&A, 286, L9

Wiklind, T., Combes, F. 1995, A\&A, 299, 382

Wiklind, T., Combes, F. 1996a, Nature, 379, 139

Wiklind, T., Combes, F. 1996b, A\&A, 315, 86

Wiklind, T., Combes, F. 1998, ApJ, 500, 129

Wilson, T. L., Matteucci, F. 1992, A\&AR, 4, 1 